\title{
Autonomous Decentralized Control of Traffic Signals that can Adapt to Changes in Traffic
}

\author{
Takeshi Kano $^{1}$. Yuki Sugiyama ${ }^{2}$. Akio Ishiguro' \\ ${ }^{1}$ Research Institute of Electrical Communication, Tohoku University, Sendai, Japan, \\ E-mail: tkano@riec.tohoku.ac.jp \\ 2 Department of Complex Systems Science, Nagoya University, Nagoya, Japan
}

Received: 13 January 2016 / Accepted: 10 October 2016

DOI: $10.17815 / C D .2016 .5$

\begin{abstract}
A major challenge for traffic signal control is adapting to unpredictable changes in traffic. To address this issue, we propose an autonomous decentralized control scheme for traffic signals that is based on physics. More specifically, "virtual impulses" given by red signals or preceding cars, which are defined in a similar manner as the impulses generally used in physics, are calculated at each traffic signal by using an optimal velocity model, and traffic signals are switched to reduce these virtual impulses. We performed simulations under various traffic conditions, and the results showed that the proposed control scheme works adaptively and resiliently in response to each set of circumstances. Thus, the virtual impulse can be a key physical quantity for designing adaptive traffic systems.
\end{abstract}

Keywords Traffic signal control $\cdot$ decentralized control $\cdot$ virtual impulse

\section{Introduction}

Traffic systems are indispensable both in industries and our daily lives. Owing to the recent increase in the number of cars, traffic jams often occur in urban areas. This causes economic and environmental problems. The development of traffic signal control methodologies is one of the most important issues for solving this problem. Traffic jams can be reduced by adequate control of traffic signals.

In designing traffic signal controls, it is important to ensure adaptability to changes in the amount of traffic because real traffic varies over time and is affected by unpredictable circumstances, e.g., traffic accidents. Accordingly, many researchers have attempted to 
address this issue [1-30]. However, solving such an adaptation problem is not an easy task, and even control schemes currently used in real traffic, such as the split-cycle offset optimization technique (SCOOT) [1] and Sydney coordinated adaptive traffic system (SCATS) [2] are not fully adaptable.

Autonomous decentralized control, in which adaptive and resilient macroscopic behavior emerges through the coordination of simple individual components, may be the key to adapting to unpredictable changes in the amount of traffic. Thus, several decentralized control schemes have been proposed so far [10-29]. A typical example is the selforganizing traffic signal (SOTL) method [10-19], where each traffic signal is controlled by several local rules such that the traffic signals can immediately adapt to the current situation. Other examples of decentralized control schemes are based on coupled oscillators [20-22, 29], neural networks [23], near-future prediction [28], and so on. These control schemes enable traffic signals to adapt to changes in the amount of traffic, yet there still remains room for further improvement.

In this study, we developed a physics-based autonomous decentralized control scheme for traffic signals to further increase the adaptability. More specifically, we defined a "virtual impulse", which is a quantity similar to the impulses generally used in physics, and used it to evaluate the switch timing of each traffic signal. We performed simulations to demonstrate that the proposed control scheme works well under various traffic conditions. Thus, we believe that our physics-based concept can form a basis for the design of adaptive traffic systems.

\section{Model}

Fig. 1 shows a schematic of the model. Single-lane and bidirectional roads that run southnorth and east-west are aligned equidistant in a $L \times L$ square lattice. We assumed that the lane width can be neglected. Traffic signals are present at all $M \times M$ intersections. Thus, the distance between neighboring traffic signals $l$ is given by $l=L /(M+1)$. The traffic signal that is $i$ th from the west and $j$ th from the south is denoted by $S_{i j}(i, j=$ $1,2, \cdots, M)$. Cars appear on the lanes at the lattice edge with the probability $p_{i^{\prime}, j^{\prime}}$ for each time interval $\tau$ and disappear when they get out of the lattice, where $i^{\prime}$ is the lane number and $j^{\prime}=s, n, w, e$ with $s, n, w$, and $e$ denoting lanes from the south, north, west, and east, respectively. However, we set an upper limit to the number of cars in each lane, defined as $N$, to prevent divergence of the simulation, and cars do not appear on lanes with $N$ cars. Cars do not turn right or left. We did not consider the effect of pedestrians or vehicles other than cars.

The motion of the cars was modeled by using the optimal velocity model [31]. The time evolution of the velocity of the $i$ th car $v_{i}$ can be described by

$$
\dot{v}_{i}=a_{i}\left\{V\left(\Delta x_{i}\right)-v_{i}\right\}
$$

where $\Delta x_{i}$ is the distance from the preceding car or red signal (whichever is smaller), $V\left(\Delta x_{i}\right)$ is a function that determines the desired velocity, and $a_{i}$ is a parameter whose inverse determines the time scale for the convergence to the desired velocity. The functional 
form of $V\left(\Delta x_{i}\right)$ was determined according to previous works [8,31,32] and is described by

$$
\left.V\left(\Delta x_{i}\right)=v_{0}\left[\tanh \left\{\kappa\left(\Delta x_{i}-d\right)\right\}+\tanh (\kappa d)\right\}\right],
$$

where $v_{0}$ is related to the maximum velocity allowed, $d$ determines the inflection point of the desired velocity curve, and $\kappa$ denotes the steepness of the desired velocity curve around the inflection point.

Each traffic signal has two states: "green for east-west road and red for south-north road" and "red for east-west road and green for south-north road." Thus, no yellow signal is assumed. The two states switch with each other via the clearance time $\delta t$, wherein both signals remain red; this clearance time is determined in real traffic by considering the safety of drivers. We assumed that each traffic signal has sensors that can detect the position and velocity of cars within the distance $l$ from the traffic signal.

The traffic signal control consists of four steps: (1) detection of the car position and velocity, (2) future prediction for car motion, (3) calculation of the virtual impulse, and (4) evaluation of signal switching. These steps are repeated every time interval $t_{a}$ at each traffic signal. We explain each step in detail below.

\section{(1) Detection of car position and velocity}

We assumed that each traffic signal can detect the position and velocity of cars within the distance $l$ from itself. For example, a traffic signal at the center on top of Fig. 1 can only detect cars in the red square. With real traffic, the car position and velocity can be detected by attaching a camera to each traffic signal.

\section{(2) Future prediction for car motion}

Once the present position and velocity of cars are detected at each traffic signal in step 1 , their motion in the near future can be predicted by numerically solving the optimal velocity model. More specifically, a virtual space where the south-north and east-west roads cross at the intersection and extend infinitely is considered, and the initial position and velocity of the cars in the virtual space are determined on the basis of sensor data obtained in step 1 . Then, the motion of each car in the near future is predicted by simulating Eq. 1 and 2. This simulation is performed until $t_{\text {virtual }}=T$, where $t_{\text {virtual }}$ is the time defined in the virtual space with $t_{\text {virtual }}=0$ being the commencement of the simulation.

Here, the car motion in the virtual space depends on the switch timing of the traffic signal. Thus, simulations are performed under the following several conditions: (i) the traffic signal is switched at $t_{\text {virtual }}=0$, (ii) the traffic signal is not switched during the period $T$, and (iii) the traffic signal is switched at $t_{\text {virtual }}=n t_{b}$, where $t_{b}$ is a positive constant and $n$ is a positive integer that satisfies $n t_{b}<T-\delta t$. The data obtained for each case are used in the next step. 


\section{(3) Calculation of the virtual impulse}

To quantitatively evaluate the predicted car motion simulated in step 2, we define a "virtual impulse" here. This quantity characterizes "to what extent cars slow down owing to the existence of their preceding cars or red signals" and is defined as follows. From Eq. 1, the equation of motion for the $i$ th car is described by

$$
\begin{aligned}
m_{i} \dot{v}_{i} & =m_{i} a_{i}\left\{V\left(\Delta x_{i}\right)-v_{i}\right\} \\
& =c\left\{V\left(\Delta x_{i}\right)-v_{i}\right\}
\end{aligned}
$$

where $m_{i}$ is the mass of the $i$ th car. We assumed that $a_{i}=c / m_{i}$, where $c$ is a positive constant because it is natural to consider inertia to apply with increasing mass. For comparison, the equation of motion when there are no preceding cars or red signals is given by

$$
m_{i} \dot{v}_{i}=c\left\{V(\infty)-v_{i}\right\} .
$$

Let $F_{i}$ be defined as the difference between the right-hand sides of Eq. 4 and 3:

$$
F_{i}=c\left\{V(\infty)-V\left(\Delta x_{i}\right)\right\}
$$

If the force acting on the $i$ th car in Eq. 4 is taken as a reference, $F_{i}$ can be interpreted as the virtual braking force asserted by the preceding car or red signal. The virtual impulse $I$ is defined by integrating $F_{i}$ over time and taking the summation for all cars in the virtual space:

$$
I=\sum_{i} \int_{0}^{T} F_{i} \mathrm{~d} t .
$$

Thus, the virtual impulse $I$ becomes large when cars have to slow down for a long time, e.g., a long queue exists behind the traffic signal. The virtual impulse is calculated for each condition, i.e., conditions (i)-(iii) in step 2, and the virtual impulse for condition $X$ is denoted by $I_{X}$.

\section{(4) Evaluation of signal switching}

The values of the virtual impulses are used to determine whether or not the traffic signal switches. If the virtual impulse for case (i) $I_{(i)}$ is the smallest among all cases, the future prediction suggests that the traffic signal should switch at present. Hence, the traffic signal switches. If this condition is not satisfied, the signal does not switch and waits until the next cycle. 
1) Detection of car position and velocity

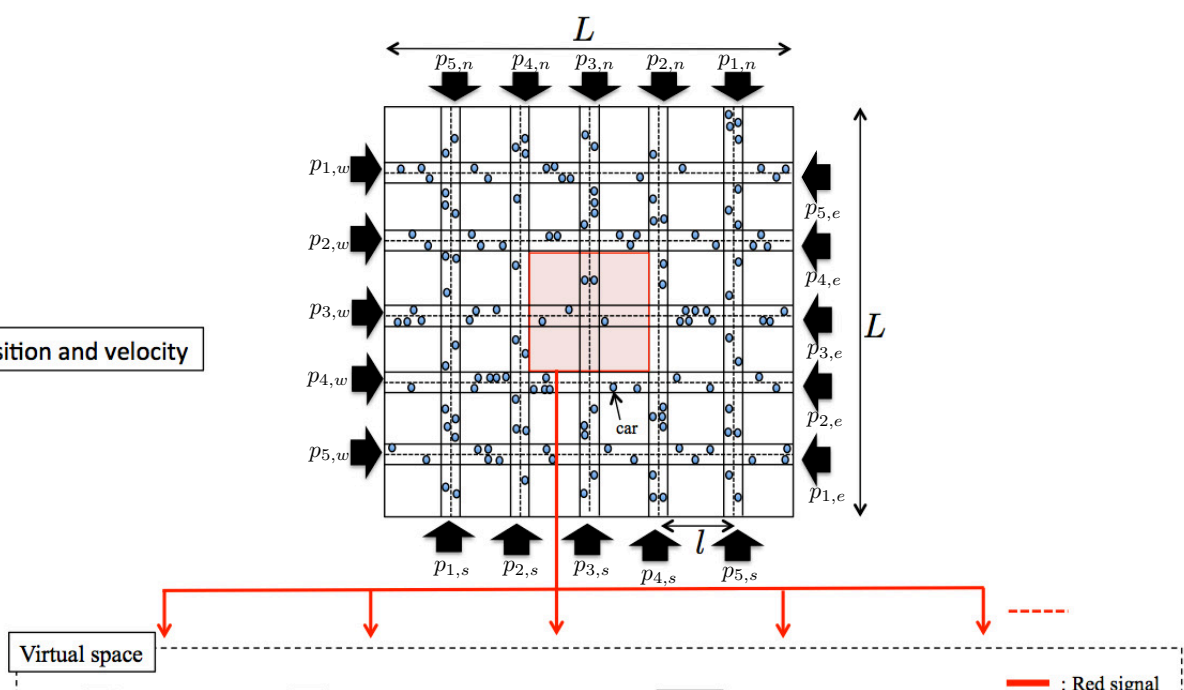

2) Future prediction
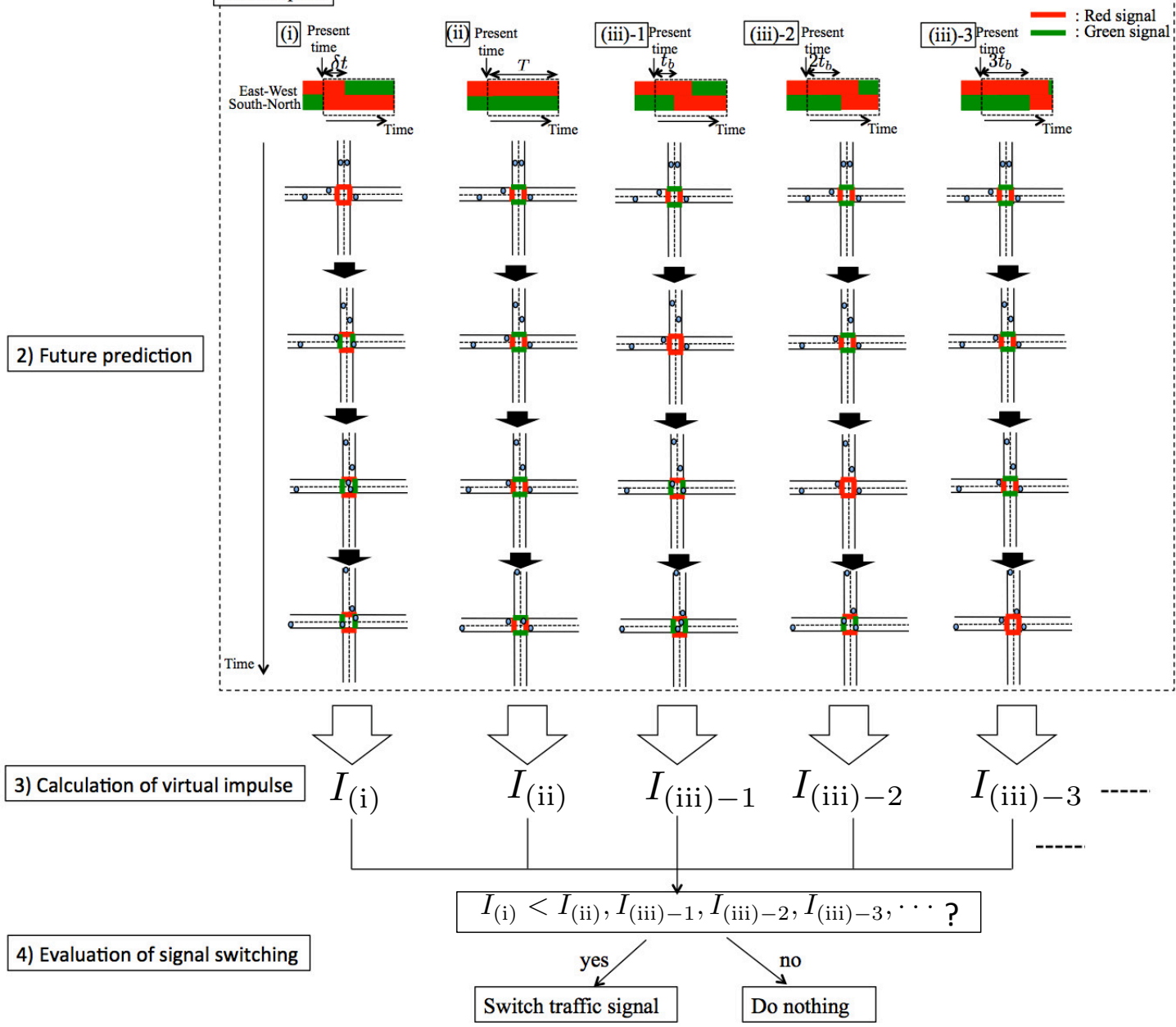

Figure 1 Overview of the model and proposed control scheme. The top figure shows the road configuration considered in the model. Particles denote cars. The process of the proposed scheme can be explained by taking the traffic signal at the center intersection as an example. First, the traffic signal detects the position and velocity of cars within the red area. Second, the motion of detected cars is predicted by performing simulations over the duration $T$ in a virtual space (dotted square). Several patterns of signal switching (patterns (i), (ii), (iii), etc.) are examined. Then, virtual impulses are calculated; on this basis, the signal switching is evaluated. 


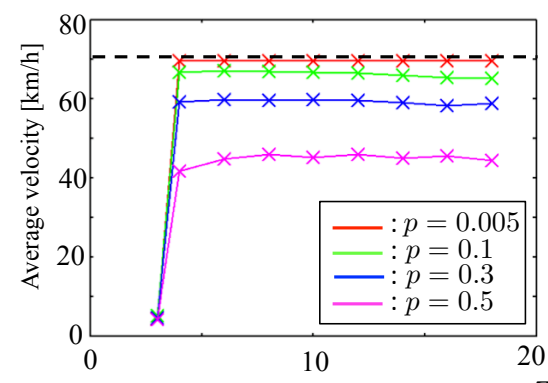

(a)

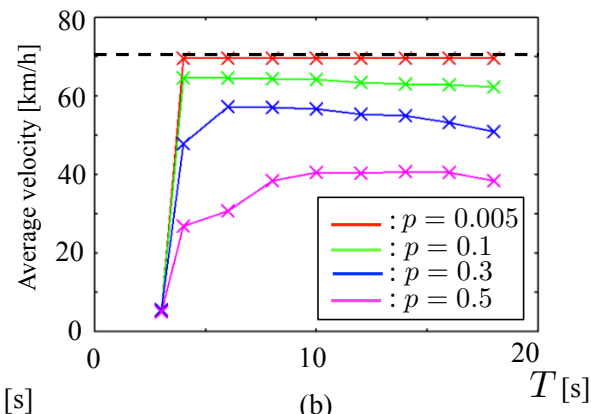

(b)

Figure 2 Average velocity in the proposed scheme when $T$ and $p$ are varied: (a) unidirectional flow, i.e., cars appear only from west and south, and (b) bidirectional flow, i.e., cars appear from all directions. The dashed line indicates the maximum velocity $V(\infty)$.

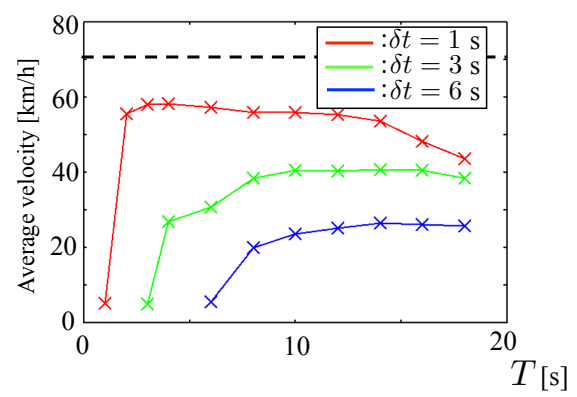

Figure 3 Average velocity in the proposed scheme when $\delta t$ and $T$ are varied. Simulations were performed for the bidirectional flow case. The dashed line indicates the maximum velocity $V(\infty)$.

\section{Simulation}

We performed simulations to test the proposed control scheme. The initial states of the traffic signals were set to be random (except for control scheme 2 described below). The initial velocity of the cars was set to zero. We investigated the cases for unidirectional flow-i.e., cars appeared either from the west or south $\left(p_{i^{\prime} w} \neq 0, p_{i^{\prime} s} \neq 0\right.$, and $p_{i^{\prime} e}=p_{i^{\prime} n}=$ $0)$ - and bidirectional flow-i.e., cars appeared from all directions $\left(p_{i^{\prime} w} \neq 0, p_{i^{\prime} e} \neq 0\right.$, $p_{i^{\prime} s} \neq 0$, and $p_{i^{\prime} n} \neq 0$ ). The performance of the control scheme was evaluated in terms of the average velocity of all cars in the lattice over the period of $400 \mathrm{~s}$. All of the simulations had the following parameter values in common: $a_{i}=1.5 \mathrm{~s}^{-1}, v_{0}=10 \mathrm{~ms}^{-1}$, $\kappa=0.1 \mathrm{~m}^{-1}, d=20 \mathrm{~m}, \tau=2 \mathrm{~s}$, and $N=100$. The time step was set to $0.02 \mathrm{~s}$. Tab. 1 lists the other parameter values used in each experiment. Here, $n_{\text {init,w }}, n_{\text {init,e }}, n_{\text {init,s }}$, and $n_{\text {init,n }}$ denote the numbers of cars initially in lanes running from the west, east, south, and north, respectively. Hereafter, we refer to each experiment by the numbers given in Tab. 1 . 


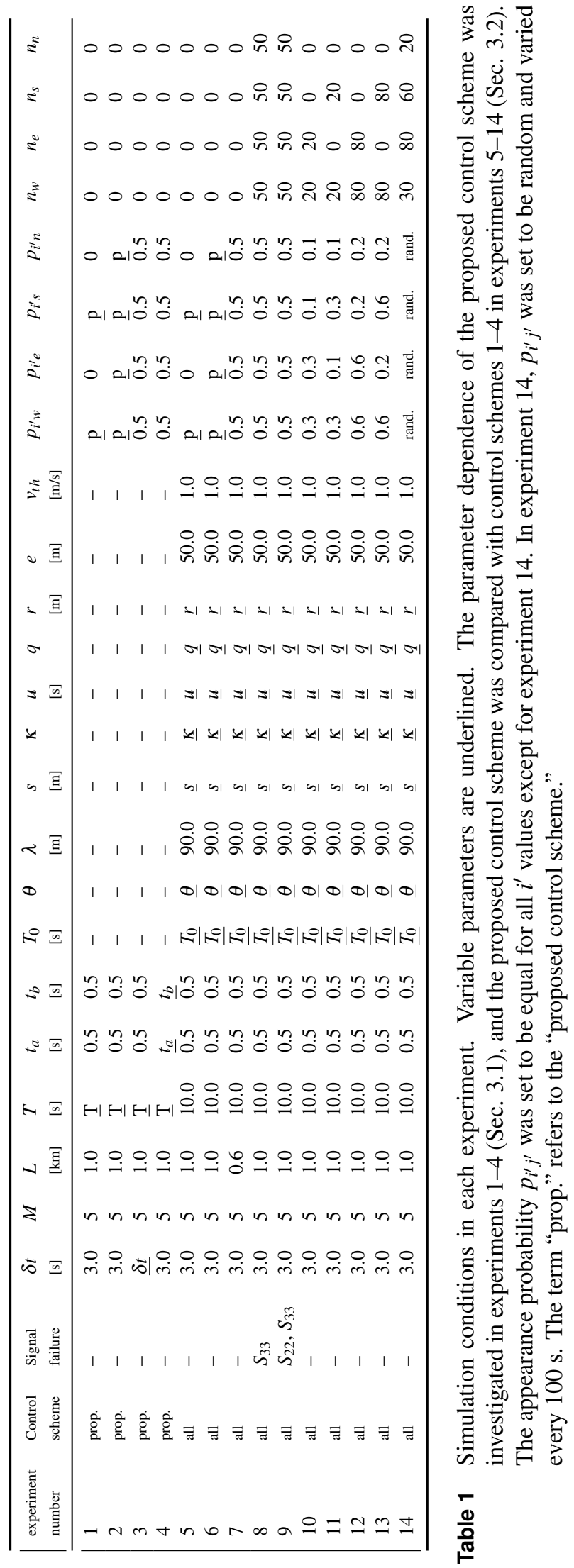



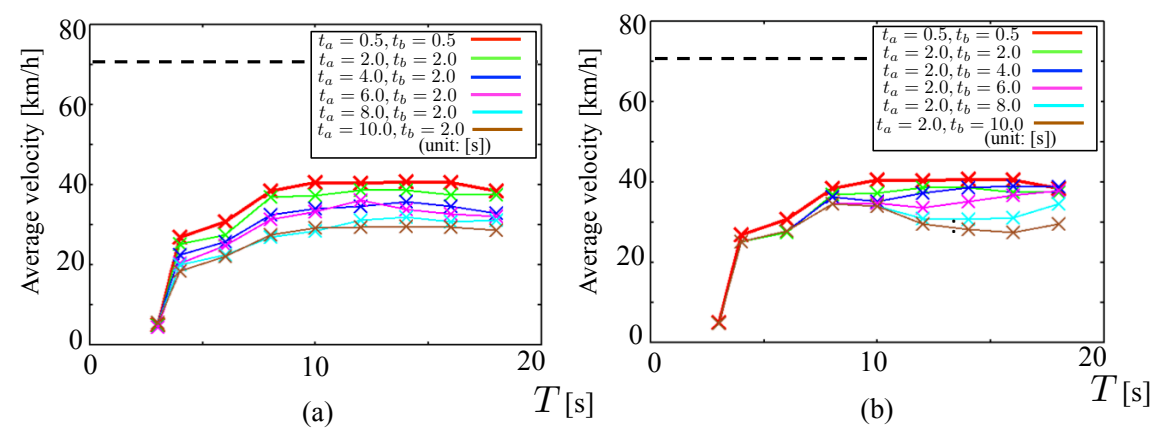

Figure 4 Simulation results when (a) $t_{b}$ was varied with $t_{a}=2.0 \mathrm{~s}$ and (b) $t_{a}$ was varied with $t_{b}=2.0 \mathrm{~s}$. For comparison, the results for $t_{a}=0.5 \mathrm{~s}$ and $t_{b}=0.5 \mathrm{~s}$ are also shown (bold line). Simulations were performed for the bidirectional flow case. The dashed line indicates the maximum velocity $V(\infty)$.

\subsection{Parameter dependence in the proposed control scheme}

First, we changed several parameters to investigate the characteristics of the proposed control scheme. Fig. 2 shows the result when the appearance probability of cars $p_{i^{\prime}, j^{\prime}}$ and the duration of future prediction $T$ were changed with the other parameters fixed (experiments 1 and 2). We found that the average velocity tended to be high at $T=6-14$ $\mathrm{s}$ for all $p_{i^{\prime}, j^{\prime}}$. This was almost the same as the characteristic time of $8.49 \mathrm{~s}$, which was derived by dividing the distance between adjacent traffic signals $l$ by the maximal velocity of the cars $V(\infty)$. Fig. 3 shows the result when the clearance time $\delta t$ and duration of future prediction $T$ were varied in the case of bidirectional flow (experiment 3 ). The average velocity generally decreased with increasing $\delta t$. The average velocity was extremely low when $T=\delta t$. Further, the value of $T$ that maximized the average velocity increased with $\delta t$.

These results can be explained as follows. When $T \leq \delta t$, the traffic signal rarely satisfied the switching condition $I_{(\mathrm{i})}<I_{(\mathrm{ii})}, I_{(\mathrm{iii})-1}, I_{(\mathrm{iii})-2}, I_{(\mathrm{iii})-3} \cdots$. This is because both the east-west and south-north signals in the virtual space remained red for case (i), i.e., the traffic signal switched at $t_{\text {virtual }}=0$. On the other hand, the prediction in the virtual space deviated from the real behavior when $T$ was considerably longer than the characteristic time $l / V(\infty)$. Thus, $T$ should be carefully determined on the basis of the characteristic time $l / V(\infty)$ and clearance time $\delta t$.

Finally, we examined the effect of the sampling intervals $t_{a}$ and $t_{b}$ (experiment 4). Fig. 4 shows the result. The average velocity decreased as $t_{a}$ and $t_{b}$ increased. However, the decrease in the average velocity was not very large when $t_{a}$ and $t_{b}$ were not longer than the characteristic time $8.49 \mathrm{~s}$. Thus, the sampling interval does not need to be extremely short compared with the characteristic time. Hence, the calculation cost for the future prediction process can be kept low. 

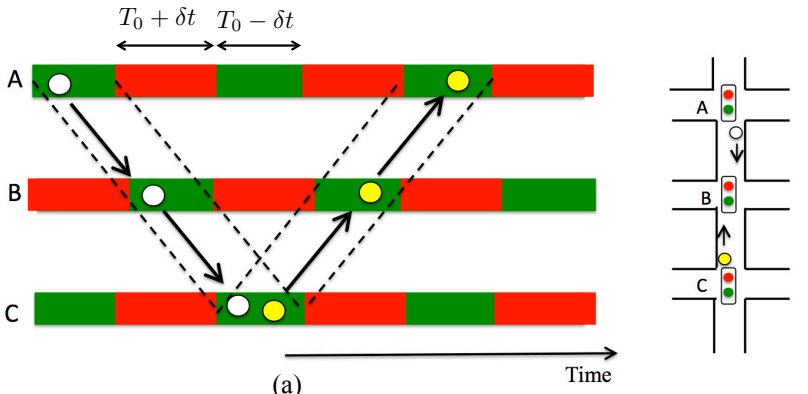

(a)
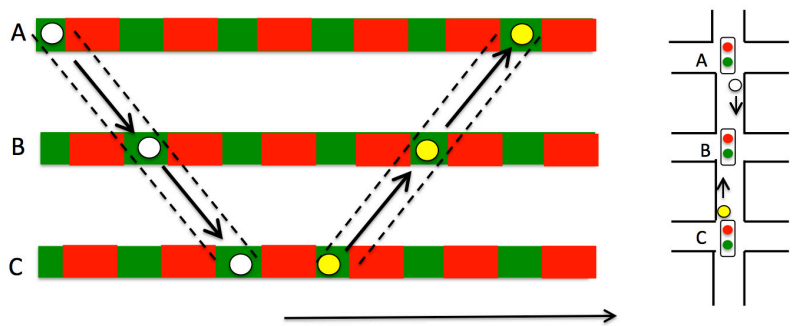

(b)

Figure 5 Schematic of the green wave method when (a) $k=1$ and (b) $k=2$. Suppose that traffic signals $\mathrm{A}, \mathrm{B}$, and $\mathrm{C}$ are aligned sequentially, as shown in the right schematic. The color bars in the left schematic denote the state of the traffic signals. Both cars moving from A to $\mathrm{B}$ to $\mathrm{C}$ (white circle) and from $\mathrm{C}$ to $\mathrm{B}$ to $\mathrm{A}$ (yellow circle) are not trapped by the red signal as long as they are moving at the maximum velocity $V(\infty)$.

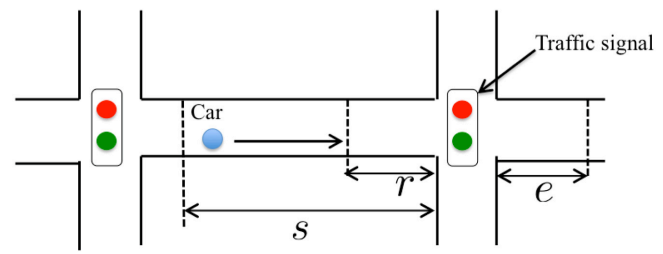

Figure 6 Definition of parameters for the SOTL method.
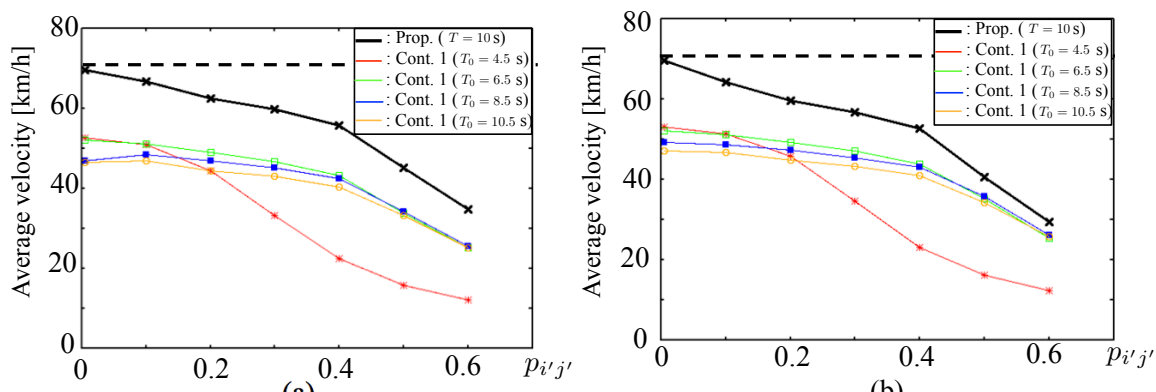

(a)

(b)

Figure 7 Comparison between the proposed control scheme and control scheme 1: (a) unidirectional flow, i.e., cars appear only from west and south (experiment 5), and (b) bidirectional flow, i.e., cars appear from all directions (experiment 6). The dashed line indicates the maximum velocity $V(\infty)$. The terms "prop." and "Cont. 1" refer to "proposed control scheme" and "control scheme 1, , respectively. 


\subsection{Comparison with other control schemes}

Next, we compared the proposed control scheme with the following previously proposed control schemes:

Control scheme 1: Fixed cycle length with random offset

Traffic signals are switched for every period $T_{0}$. The offsets between adjacent traffic signals are set to be random. This is an open-loop control, and no sensory feedback is implemented.

Control scheme 2: Green wave method [8, 10-14]

The green wave method is the same as control scheme 1 in that traffic signals are switched for every period $T_{0}$ without any sensory feedback. However, the offset is defined so that cars moving from south to north and from west to east at their maximum velocity are not trapped by red signals. More specifically, traffic signal $S_{i j}$ switches when the duration $(i+j-2) l / V(\infty)$ passes after $S_{11}$ has switched. Note that cars moving from north to south and from east to west can be trapped at each traffic signal; this can be avoided by properly choosing $T_{0}$. In fact, when $T_{0}=l /(V(\infty) k)(k=1,2, \cdots)$, cars moving at the maximum velocity $V(\infty)$ are not trapped at traffic signals, as shown in Fig. 5.

Control scheme 3: control scheme proposed by Suzuki et al. [24]

When the traffic signal along the south-north road is red, the traffic signal switches if $N_{s}+N_{n}-N_{e}-N_{w}>\theta$ is satisfied, where $\theta$ is a positive integer and $N_{e}, N_{w}, N_{s}$, and $N_{n}$ are the numbers of cars that approach the traffic signal from the east, west, south, and north, respectively, within a fixed distance $\lambda$ from the traffic signal. When the traffic signal along the east-west road is red, the traffic signal switches if $N_{e}+N_{w}-N_{s}-N_{n}>\theta$ is satisfied.

Control scheme 4: Self-organizing traffic light (SOTL) method [10-19]

The SOTL method was first proposed by Gershenson et al. in 2005 [10] and extended in later studies [11-14]. Here, we adopted the extended version, which consists of the following six rules (the original version [10] consists of only rules $1-3)$ :

Rule 1 At every time step, add to a counter the number of vehicles approaching or waiting at a red light within the distance $s$ (Fig. 6). When this counter exceeds the threshold $\kappa$, switch the light. (Whenever the light switches, reset the counter to 0 .)

Rule 2 Lights must remain green for the minimum time $u$.

Rule 3 If a few vehicles ( $q$ or fewer but more than zero) are left to cross a green light at a short distance $r$ (Fig. 6), do not switch the light. 

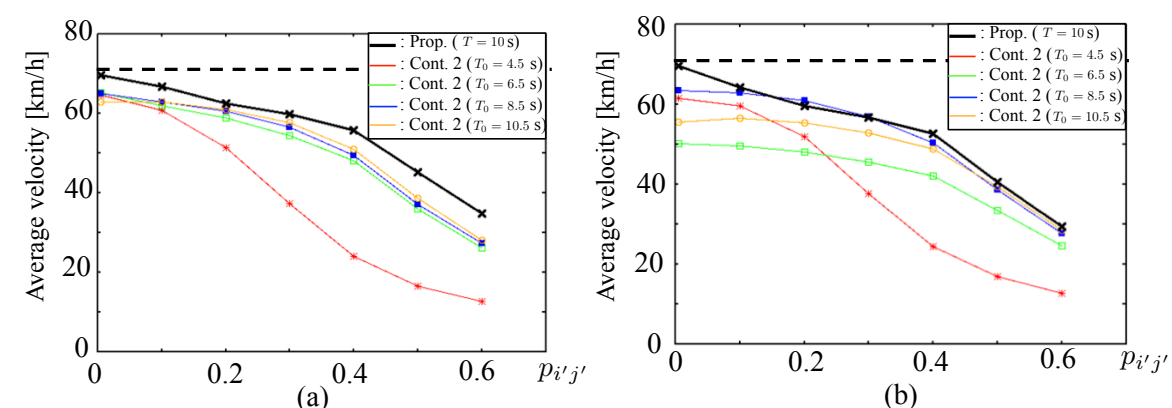

Figure 8 Comparison between the proposed control scheme and control scheme 2: (a) unidirectional flow, i.e., cars appear only from the west and south (experiment 5), and (b) bidirectional flow, i.e., cars appear from all directions (experiment 6). The dashed line indicates the maximum velocity $V(\infty)$. The terms "prop." and "Cont. 2" refer to the "proposed control scheme" and "control scheme 2," respectively.

Rule 4 If no vehicle is approaching a green light within the distance $s$ and at least one vehicle is approaching the red light within the distance $s$, then switch the light.

Rule 5 If a vehicle is stopped on the road within a short distance $e$ beyond a green traffic light (Fig. 6), then switch the light.

Rule 6 If there are vehicles stopped in both directions at a short distance $e$ beyond the intersection (Fig. 6), then switch both lights to red. Once one of the directions is free, restore the green light in that direction.

Note that, when we implemented these rules in our simulator, modification of rules 5 and 6 was inevitable because cars did not stop completely but moved slowly even when the distance from the preceding cars or red signals was short in our model (Eq. 1 and 2). Thus, we used rules $5^{\prime}$ and $6^{\prime}$ instead of rules 5 and 6 in the present study:

Rule $5^{\prime}$ If there is a decelerating vehicle whose velocity is less than $v_{t h}$ on the road within a short distance $e$ beyond a green traffic light, then switch the light.

Rule $6^{\prime}$ If there are decelerating vehicles whose velocities are less than $v_{t h}$ in both directions at a short distance $e$ beyond the intersection, then switch both lights to red. Once one of the directions is free, restore the green light in that direction.

We performed simulations of control schemes 1-4 by changing the appearance probability $p_{i^{\prime} j^{\prime}}$ for the uni- and bidirectional flow cases, and the results were compared with those for the proposed control scheme with $T=10 \mathrm{~s}$ (experiments 5 and 6). We also examined cases $(\mathrm{A})-(\mathrm{H})$ to investigate adaptability to various traffic conditions (experiments 7-14).

(A) The lattice length $L$ is small (experiment 7). 

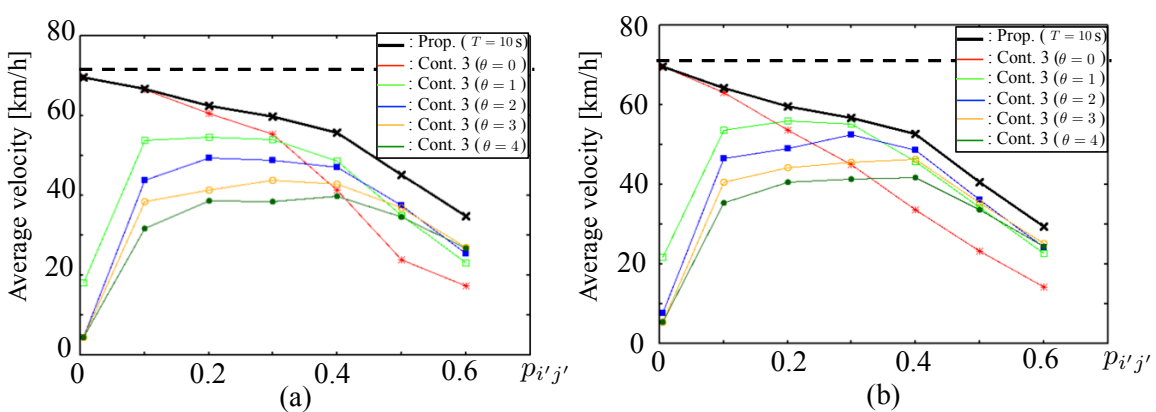

Figure 9 Comparison between the proposed control scheme and control scheme 3: (a) unidirectional flow, i.e., cars appear only from the west and south (experiment 5), and (b) bidirectional flow, i.e., cars appear from all directions (experiment 6). The dashed line indicates the maximum velocity $V(\infty)$. The terms "prop." and "Cont. 3" refer to the "proposed control scheme" and "control scheme 3," respectively.

(B) One of the traffic signals $\left(S_{33}\right)$ is broken and remains red in both the north-south and east-west directions (experiment 8).

(C) Two of the traffic signals $\left(S_{22}\right.$ and $\left.S_{33}\right)$ are broken and remain red in both the northsouth and east-west directions (experiment 9).

(D) The appearance probability of cars $p_{i^{\prime} j^{\prime}}$ for the lanes from west and east is greater than that for the other lanes $\left(p_{i^{\prime} w}=p_{i^{\prime} e}=0.3\right.$ and $\left.p_{i^{\prime} s}=p_{i^{\prime} n}=0.1\right)$ (experiment $10)$.

(E) The appearance probability of cars $p_{i^{\prime} j^{\prime}}$ for the lanes from west and south is greater than that for the other lanes $\left(p_{i^{\prime} w}=p_{i^{\prime} s}=0.3\right.$ and $\left.p_{i^{\prime} e}=p_{i^{\prime} n}=0.1\right)$ (experiment 11).

(F) The appearance probability of cars $p_{i^{\prime} j^{\prime}}$ for the lanes from west and east is greater than that for the other lanes $\left(p_{i^{\prime} w}=p_{i^{\prime} e}=0.6\right.$ and $\left.p_{i^{\prime} s}=p_{i^{\prime} n}=0.2\right)$ (experiment 12).

(G) The appearance probability of cars $p_{i^{\prime} j^{\prime}}$ for the lanes from west and east is greater than that for the other lanes $\left(p_{i^{\prime} w}=p_{i^{\prime} s}=0.6\right.$ and $\left.p_{i^{\prime} e}=p_{i^{\prime} n}=0.2\right)$ (experiment 13).

H) The appearance probability of cars $p_{i^{\prime} j^{\prime}}$ varies randomly within $[0,1.0]$ every $100 \mathrm{~s}$ (experiment 14).

The results are shown in Fig. 7 and 11. The average velocity for the proposed control scheme was generally higher than those for control schemes 1-4. Control schemes 1-4 were comparable with the proposed control scheme in some cases, but the parameters had to be finely tuned to fit each traffic condition. In contrast, the proposed control scheme 

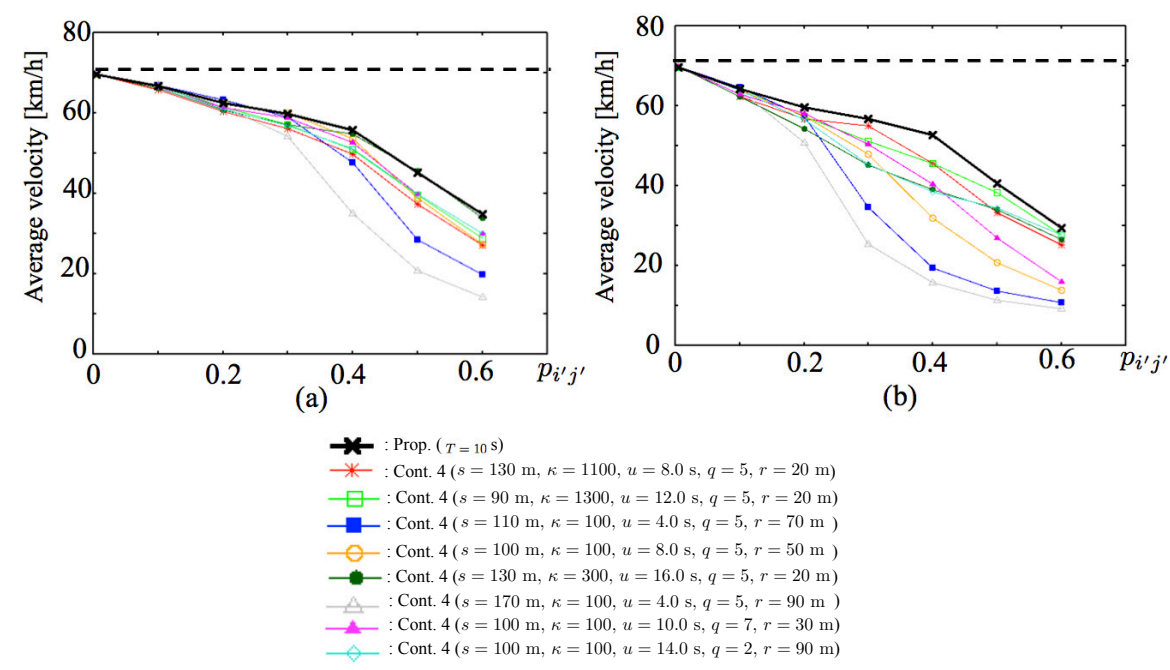

Figure 10 Comparison between the proposed control scheme and control scheme 4: (a) unidirectional flow, i.e., cars appear only from the west and south (experiment 5), and (b) bidirectional flow, i.e., cars appear from all directions (experiment 6). The dashed line indicates the maximum velocity $V(\infty)$. The terms "prop." and "Cont. 4" refer to the "proposed control scheme" and "control scheme 4," respectively.

could adapt to various traffic conditions without changing any parameter (Movies 1-16: parameter values are given in Supplementary Tab. 1) $)^{1}$.

Now, we look at each control scheme in detail. As shown in Fig. 7 (experiments 5 and 6 ), the performance of control scheme 1 depended on the cycle length $T_{0}$. When $p_{i^{\prime} j^{\prime}}$ was small, the average velocity increased as $T_{0}$ decreased. This is because cars need not wait for a long time at each intersection owing to the frequent switching of traffic signals when $T_{0}$ is small (Movie 17). On the other hand, when $p_{i^{\prime} j^{\prime}}$ was large, the opposite tendency was obtained, i.e., the average velocity decreased with $T_{0}$. This is because the frequent switching of traffic signals when $T_{0}$ is small hinders traffic flow owing to the existence of a clearance time (Movie 18). In all cases, the average velocity for control scheme 1 was lower than that of the proposed control scheme, which indicates that control scheme 1 lacks adaptability to changes in traffic.

Control scheme 2 generally performed better than control scheme 1 because traffic signals switched in accordance with the approach of cars when they moved at their maximum velocity. The average velocity for unidirectional flow (experiment 5) was comparable with that of the proposed control scheme except when $T_{0}$ was small and $p_{i^{\prime} j^{\prime}}$ was large (Fig. 8(a)). For small $T_{0}$ and large $p_{i^{\prime} j^{\prime}}$, a long queue formed before the first traffic signal in each lane owing to the frequent switching, although cars could move at almost the maximum velocity once they passed the first signal (Movie 19). This tendency also applied in the case of a bidirectional flow (Fig. 8(b), experiment 6).

The average velocity for the bidirectional flow was comparable with that of the pro-

\footnotetext{
${ }^{1}$ Supplementary movies and a corresponding table with the simulation conditions can be found at http://collective-dynamics.eu/index.php/cod/article/view/A5
} 

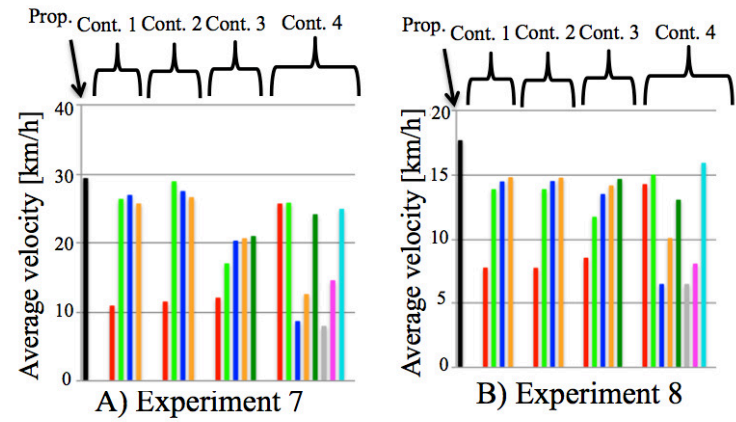

B) Experiment 8

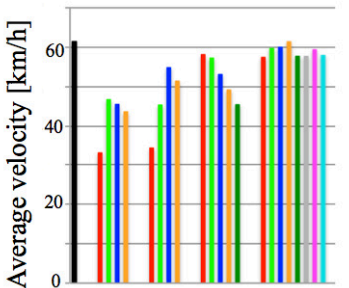

D) Experiment 10

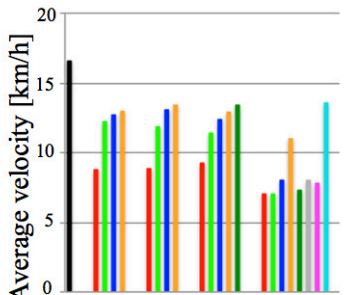

G) Experiment 13

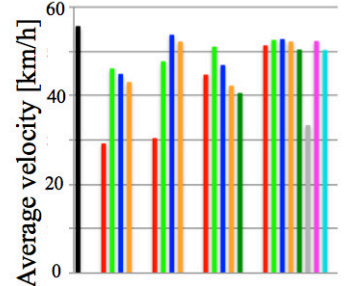

E) Experiment 11

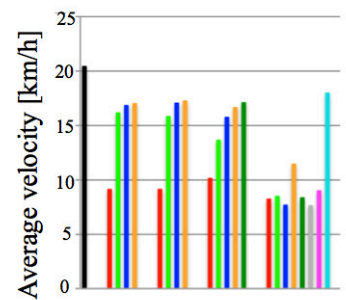

H) Experiment 14

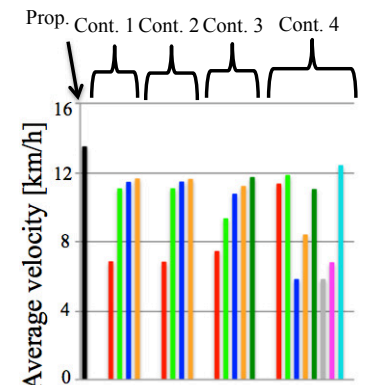

C) Experiment 9

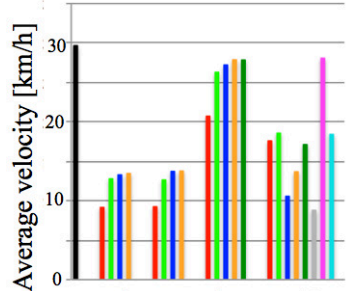

F) Experiment 12

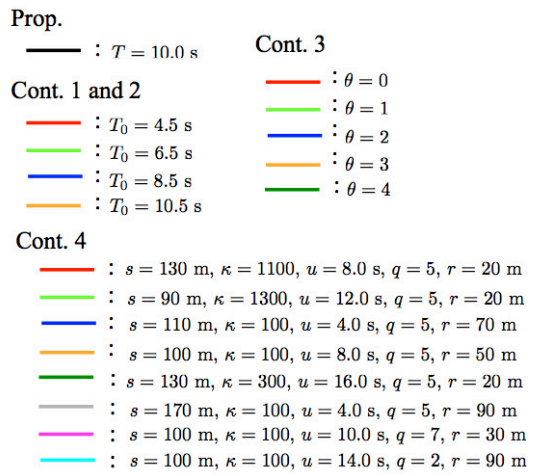

Figure 11 Results for experiments 7-14. The results for the proposed control scheme with $T=10 \mathrm{~s}$ and control schemes 1-4 with various parameters are shown. The terms "prop." and "Cont. X" refer to the "proposed control scheme" and "control scheme X," respectively.

posed control scheme when $T_{0}=8.5 \mathrm{~s}$ but was low when $T_{0}=6.5 \mathrm{~s}$ (Fig. 8(b)). This is because cars moving from east to west and from north to south at the maximum velocity need not stop when $T_{0}=8.5 \mathrm{~s}$, which is almost the same as $l / V(\infty)(=8.49 \mathrm{~s})$ (Fig. 5, Movie 20), while they have to stop at each traffic signal when $T_{0}=6.5 \mathrm{~s}$ (Movie 21).

Thus, control scheme 2 with $T_{0}=8.5 \mathrm{~s}$ worked well in experiments 5 and 6 where $p_{i^{\prime} j^{\prime}}$ was varied for uni- and bidirectional flows. However, the performance was still worse than the proposed control scheme under several conditions (Fig. 11, experiments 7-14), which suggests that control scheme 2 still lacks adaptability to changes in traffic.

The performance of control scheme 3 depended on the parameter $\theta$ (Fig. 9). When $\theta$ was small, traffic signals switched with the arrival of a small number of cars, which is beneficial when $p_{i^{\prime} j^{\prime}}$ is small. However, frequent switching caused by small $\theta$ hindered the traffic flow when $p_{i^{\prime} j^{\prime}}$ was large (Movie 22). On the other hand, when $\theta$ was large, traffic signals could not switch until a sufficient number of cars arrived at the intersections. 
As a consequence, the average velocity was slow for small $p_{i^{\prime} j^{\prime}}$ (Movie 23). However, a large $\theta$ was beneficial when $p_{i^{\prime} j^{\prime}}$ was large because frequent switching was avoided.

Control scheme 4 contained several parameters, and we performed simulations by changing them. Several sets of representative data when good performances were obtained are shown in Fig. 10 and 11. Control scheme 4 was comparable with the proposed control scheme with small $p_{i^{\prime} j^{\prime}}$ in experiments 5 and 6; rule 4 played a crucial role in the adaptation of traffic signals. However, when $p_{i^{\prime} j^{\prime}}$ was at an intermediate value or larger, the parameter values that yielded a good performance varied with the traffic conditions.

It is difficult to fully explain the relationship between the parameters and performance in control scheme 4 because the number of parameters was large. However, the tendency that the average velocity is low for small $u$ and large $p_{i^{\prime} j^{\prime}}$ was captured (Fig. 10, Movie 24, experiments 5 and 6). In this case, frequent switching of traffic signals hindered traffic flow. However, the performance at large $u$ was still worse than that of the proposed control scheme under several traffic conditions (Fig. 11, experiments 7-14) because rule 2 prevented the fast switching of traffic signals even when it was needed for adaptation.

In summary, the proposed control scheme can work well under various traffic conditions without tuning any parameter, in contrast to other methods that require parameters to be finely tuned to each traffic condition. This is because the switch timing of each traffic signal is properly determined based on the prediction of the nearby traffic flow, which can work well regardless of the traffic conditions.

\section{Conclusion}

We developed a decentralized control scheme for traffic signals that can adapt to changes in the amount of traffic. Our idea is based on physics: we defined a "virtual impulse," which is similar in concept to the impulse generally used in physics, and used it to evaluate the switch timing of each traffic signal. We performed simulations under several conditions with a simple model, and the results showed that our proposed control scheme worked well.

It is still unclear whether our proposed control scheme works better than other previous control schemes in real situations where cars turn right or left on complicated road networks; thus, this should be tested in the future. However, we believe that our control scheme can be used for a wide range of traffic conditions because the design is reasonable from a physical viewpoint. We also expect that our basic idea can be applied to the control of flow in open systems other than traffic flow, such as assembly production flow [33] and information flow [34].

Acknowledgements This work was supported by a Grant-in-Aid for Challenging Exploratory Research (No. 26630193) from the Ministry of Education, Culture, Sports, Science and Technology (MEXT), Japan. 


\section{References}

[1] P.B. Hunt, D.L. Robertson, and R.D. Bretherton: The SCOOT On-line Traffic Signal Optimization Technique. Traffic Eng. Control 23, 190-192 (1982).

[2] A.G. Sime and K. W. Dobinson: The Sydney Coordinated Adaptive Traffic (SCAT) System Philosophy and Benefits. IEEE Trans. Vehicul. Tech. VT-29, 130-137 (1980). doi:10.1109/T-VT.1980.23833

[3] M. Papageorgiou, C. Diakaki, V. Dinopoulou, A. Kotstalos, and Y. Wang: Review of Road Traffic Control Strategies. Proc. of the IEEE 91, 2043-2067 (2003). doi:10.1109/JPROC.2003.819610

[4] S. P. Shepherd: A Review of Traffic Signal Control, Working Paper 349, Institute of Transport Studies, University of Leeds, Leeds, UK (1992).

[5] J.J. Sanchez-Medina, M.J. Galan-Moreno, and E. Rubio-Royo: Traffic Signal Optimization in "La Almozara" District in Saragossa under Congestion Conditions, Using Genetic Algorithms, Traffic Microsimulation, and Cluster Computing. IEEE Trans. Intel. Transport. Sys. 11, 132-141 (2010). doi:10.1109/TITS.2009.2034383

[6] S. M. Rahman and N. T. Ratrout: Review of the Fuzzy Logic Based Approach in Traffic Signal Control: Prospects in Saudi Arabia. J. Trans. Sys. Eng. Info. Tech. 9, 58-70 (2009). doi:10.1016/S1570-6672 (08)60080-X

[7] H. Hong-Di, L. Wei-Zhen, and D. Li-Yun, An Improved Cellular Automaton Model Considering the Effect of Traffic Lights and Driving Behaviour, Chin. Phys. B, 20, 040514 (2011). doi:10.1088/1674-1056/20/4/040514

[8] M. Sasaki and T. Nagatani, Transition and Saturation of Traffic Flow Controlled by Traffic Lights, Physica A 325 (2003) 531-546 (2003). doi:10.1016/s0378-4371(03)00148-1

[9] D. Srinivasan, M.C. Choy, and R.L. Cheu: Neural Networks for Real-time Traffic Signal Control. IEEE Trans. Intel. Trans. Sys. 7 261-272 (2006). doi:10.1109/TITS.2006.874716

[10] C. Gershenson, Self-organizing Traffic Lights, Complex Systems, 16, 29-53 (2005)

[11] C. Gershenson and D.A. Rosenblueth, Self-organizing Traffic Lights at Multiple-street Intersections, Complexity, 17, 23-39 (2012). doi:10.1002/cplx.20392

[12] C. Gershenson and D.A. Rosenblueth, Modeling Self-organizing Traffic Lights with Elementary Cellular Automata, arXiv:0907.1925 (2009) 
[13] C. Gershenson and D.A. Rosenblueth, Adaptive Self-organization vs Static Optimization - A Qualitative Comparison in Traffic Light Coordination, Kybernetes, 41, 386-403 (2012) doi:10.1108/03684921211229479

[14] D. Zubillaga, G. Cruz, Luis D. Aguilar, J. Zapotécatl, N. Fernández, J. Aguilar, D.A. Rosenblueth and C. Gershenson, Measuring the Complexity of Self-Organizing Traffic Lights, Entropy 16, 2384-2407 (2014). doi: 10.3390 / e16052384

[15] J. de Gier, T.M. Garoni, and O. Rojas, Traffic Flow on Realistic Road Networks with Adaptive Traffic Lights, J. Stat. Mech.: Theory Exp. 2011, P04008 (2011). doi:10.1088/1742-5468/2011/04/P04008

[16] S.B. Cools, C. Gershenson, B. D’Hooghe, Self-organizing Traffic Lights: A Realistic Simulation, In: Advances in Applied Self-Organizing Systems (Part of the series Advanced Information and Knowledge Processing), (Ed.) M. Prokopenko (London: Springer) Chapter 3, 41-50. (2008). doi : 10 . 1007/978-1-84628-982-8_3

[17] A. Reztsov, On Self-Organising Traffic Lights Technology, Complexity (2015). doi:10.1002/cplx.21659

[18] A. Reztsov, Self-Organising Traffic Lights (SOTL) as an Upper Bound Estimate, Complex Syst. 24, 175-183 (2015). doi:10.2139/ssrn.2467948

[19] L. Zhang, T.M. Garoni, J. de Gier, A Comparative Study of Macroscopic Fundamental Diagrams of Arterial Road Networks Governed by Adaptive Traffic Signal Systems, Trans. Res. Part B, 49, 1-23 (2013). doi:10.1016/j.trb.2012.12.002

[20] K. Sekiyama, J. Nakanishi, I. Takagawa, T. Higashi, and T. Fukuda: Self-Organizing Control of Urban Traffic Signal Network. In: IEEE Int. Conf. Sys. Man. Cybern. 4, 2481-2486 (2001). doi:10.1109/ICSMC .2001.972930

[21] I. Nisikawa: Dynamics of Oscillator Network and Its Application to Offset Control of Traffic Signals. In: Proc. IEEE Int. Joint Conf. on Neural Networks 2, 1273-1277 (2004). doi:10.1109/IJCNN.2004.1380126

[22] M. Sugi, H. Yuasa, J. Ota, and T. Arai: Autonomous Decentralized Control of Traffic Signals with Closed-Loop Constraints on Offsets, In: Proc. SICE Ann. Conf., 19541960 (2003).

[23] T. Ohira, K. Inoue, and Y. Takeshima: Adaptive Traffic Control Model with Neural Network Analogy. In: Proc. Int. Conf. Neural Info. Proc. Intel. Info. Sys. (ICONIP), 939-942 (1997).

[24] H. Suzuki, J. Imura, and K. Aihara: Chaotic Ising-like Dynamics in Traffic Signals. Sci. Reports 3,1127 (2013). doi:10.1038/s rep01127 
[25] M.E. Fouladvand, M.R. Shaebani, Z. Sadjadi, Intelligent Controlling Simulation of Traffic Flow in a Small City Network, J. Phys. Soc. Japan, 73, 3209-3214 (2004). doi:10.1143/JPSJ.73.3209

[26] M.E. Fouladvand, Z. Sadjadi, M.R. Shaebani, Optimised Traffic Flow at a Single Intersection: Traffic Responsive Signalisation, J. Phys. A: Math. Gen. 37 561-576 (2004). doi:10.1088/0305-4470/37/3/002

[27] M.E. Fouladvand and M. Nematollahi, Optimization of Green-times at an Isolated Urban Crossroads, Eur. Phys. J. B 22 395-401 (2001). doi:10.1007/PL00011149

[28] S. Lämmer and D. Helbing, Self-control of Traffic Lights and Vehicle Flows in Urban Road Networks, J. Stat. Mech.: Theory Exp. 2008, P04019 (2008). doi:10.1088/1742-5468/2008/04/P04019

[29] S. Lämmer, H. Kori, K. Peters, and D. Helbing, Decentralised Control of Material for Traffic Flows in Networks Using Phase-synchronisation, Physica A, 363, 39-43 (2006). doi:10.1016/j.physa.2006.01.047

[30] R. Kutadinata, W. Moase, C. Manzie, L. Zhang, and T. Garoni, Enhancing the Performance of Existing Urban Traffic Light Control through Extremum-seeking, Transport. Res. Part C: Emerg. Tech., 62, 1-20 (2016). doi:10.1016/j.trc.2015.10.016

[31] M. Bando, K. Hasebe, A. Nakayama, A. Shibata, and Y. Sugiyama: Dynamical Model of Traffic Congestion and Numerical Simulation. Phys. Rev. E 51, 10351042 (1995). doi:10.1103/PhysRevE.51.1035

[32] M. Bando, K. Hasebe, K. Nakanishi, A. Nakayama, A. Shibata, and Y. Sugiyama: Phenomenological Study of Dynamical Model of Traffic Flow. Journal de Physique I, EDP Sciences, 5, 1389-1399, (1995). doi:10.1051/jp1:1995206

[33] T. Ezaki, D. Yanagisawa, and K. Nishinari: Dynamics of Assembly Production Flow. Physica A 427, 62-74 (2015). doi:10.1016/j.physa.2015.02.005

[34] M. Gerla and L. Kleinrock: Flow Control: A Comparative Survey. IEEE Trans. Comm., COM-28, 553-573 (1980). doi:10.1109/TCOM.1980.1094691 